\title{
Prevalence and characteristics of the aberrant anterior tibial artery: a single- center magnetic resonance imaging study and scoping review
}

\author{
Julio Marin-Concha ${ }^{1,2}$, Pablo Rengifo ${ }^{1}$, Pedro Tapia' ${ }^{1}$, Daniel Kaiser ${ }^{3}$ and Timo Siepmann ${ }^{2,4^{*}}$
}

\begin{abstract}
Background: Planning surgical procedures of the lower leg benefits from considering the possibility of an aberrant anterior tibial artery (AATA), but previously published data on the frequency of this anatomic variant shows heterogeneity. We assessed the prevalence of AATA in a Latin American cohort using magnetic resonance imaging (MRI) and compared these with other studies reported in the literature.

Methods: We retrospectively included consecutive patients who had undergone multiplanar knee MRI at a radiology department in Lima, Peru. The MRI protocol included coronal T1 weighted, axial, sagittal and coronal proton density fat-saturated (PDFS) and sagittal T2 weighted images.

Two experienced radiologists assessed all images and were blinded to each other's findings. The frequency of the AATA was compared to previous cohorts. A scoping review was undertaken to provide an overview of previously published data on the prevalence of ATAA.

Results: We analyzed 280 knee MRI examinations of 253 patients (median age 41 years (IQR 31-52), 53.8\% male). The aberrant anterior tibial artery variant was present in 8 of 280 (2.9\%) evaluated knees, resulting in a prevalence of 3.2\% in our study population. The PDFS sequence in the axial or sagittal orientation was most effective to identify AATA. The frequency of AATA in the reviewed literature using different radiological modalities ranged from 0.4 to $6 \%$ (median $1 \%$, IQR (0.5-2.3\%).
\end{abstract}

Conclusions: The AATA is a frequent vascular variant that can be detected by MRI in the preparation of invasive interventions of the lower leg.

Keywords: Popliteal artery, Aberrant anterior tibial artery, MRI, Anatomical variations, Knee

\section{Background}

The anatomic variations of popliteal artery terminal branching are relatively common with a prevelance of around $10 \%$ of limbs and should be considered by

*Correspondence: timo.siepmann@uniklinikum-dresden.de

${ }^{4}$ Department of Neurology, University Hospital Carl Gustav Carus, Technische Universität Dresden, Dresden, Germany

Full list of author information is available at the end of the article radiologists and surgeons [1]. Vascular injuries during invasive procedures are rare but can cause serious complications such as arterial transection, pseudoaneuryms and limb loss [2-4]. The popliteal artery is the continuation of the femoral artery after it passes through the adductor hiatus, and it normally ends at the inferior border of the popliteus muscle where it splits into the anterior tibial artery and the tibioperoneal trunk [5]. Previous research has reported different types of anatomical original author(s) and the source, provide a link to the Creative Commons licence, and indicate if changes were made. The images or other third party material in this article are included in the article's Creative Commons licence, unless indicated otherwise in a credit line to the material. If material is not included in the article's Creative Commons licence and your intended use is not permitted by statutory regulation or exceeds the permitted use, you will need to obtain permission directly from the copyright holder. To view a copy of this licence, visit http://creativecommons.org/licenses/by/4.0/. The Creative Commons Public Domain Dedication waiver (http://creativeco mmons.org/publicdomain/zero/1.0/) applies to the data made available in this article, unless otherwise stated in a credit line to the data. 
variations of popliteal terminal branching such as the high origin of the anterior tibial artery, which manifests when the vessels split above the knee joint or at the upper part of the popliteus muscle [6]. The aberrant anterior tibial artery (AATA) is a subtype variant in which the artery courses between the popliteal muscle and the tibial cortex (Figs. 1 and 2). This variant has been reported in autopsy, angiography, ultrasound and computed tomography (CT) angiography studies [6-8]. However, data on frequency vary, and the capacity of magnetic resonance imaging (MRI) to detect this variant is not widely reported [9]. Using MRI to assess AATA may provide an adequate report for the referral physician especially if surgery is planned. We aimed to assess the frequency and magnetic resonance characteristics of the AATA in a Latin American cohort and compared our findings with previous research done with other imaging modalities by a scoping review.

\section{Methods}

\section{Subjects, protocol}

We evaluated MRI examinations of the knee of consecutive patients who had been assessed at our radiology department in Lima, Peru (Radiology Department Clínica SANNA, San Borja, Lima, Peru) from October 2018 to December 2018. To enhance external validity we did not restrict any indications of MRI in the selection of subjects. Our exclusion criteria were an inadequate knee MRI evaluation due to motion artifact or theinability of the patient to complete the test. The need for ethics approval and consent was waived by the Institutional Review Boards of Clinica San Borja and the Universidad Peruana Cayetano Heredia (19-6681) due to the retrospective nature of the study. We obtained permission from Clinica SANNA, San Borja to get access to the clinical and imaging patient data. Patient characteristics were recorded according to the clinical history. a

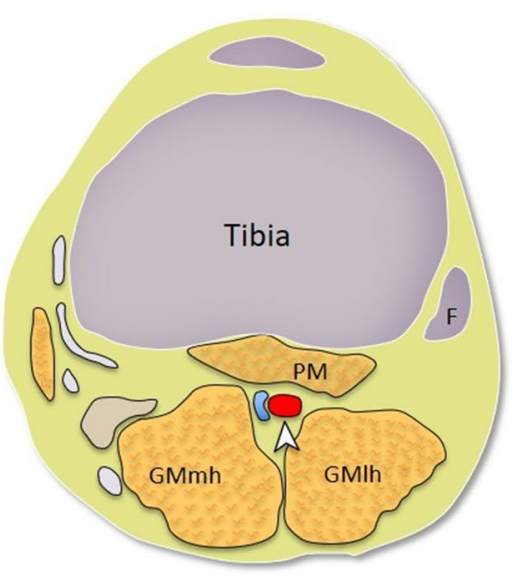

C

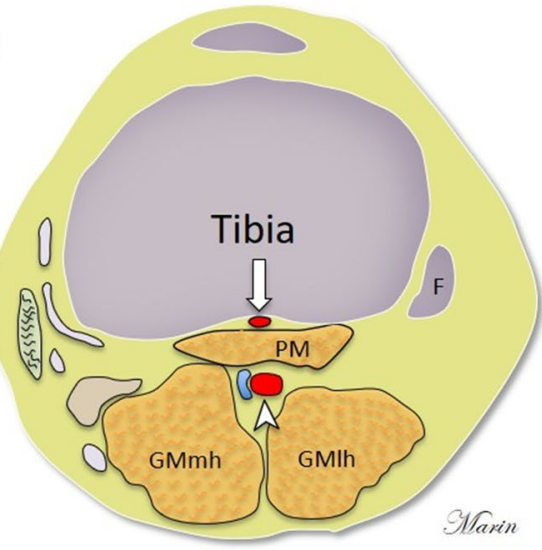

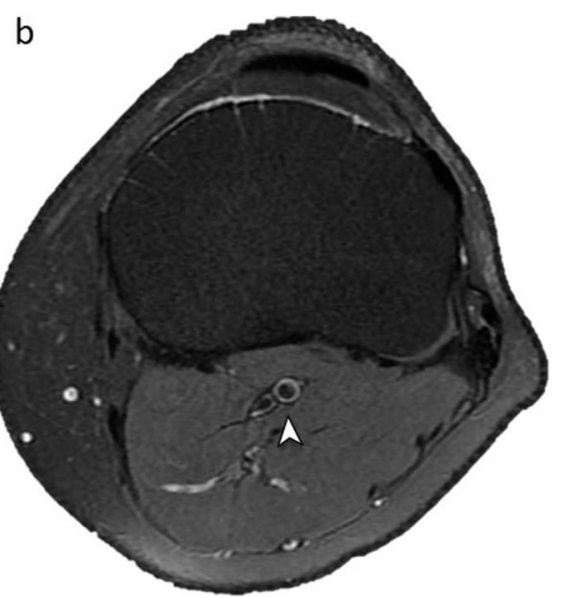

d

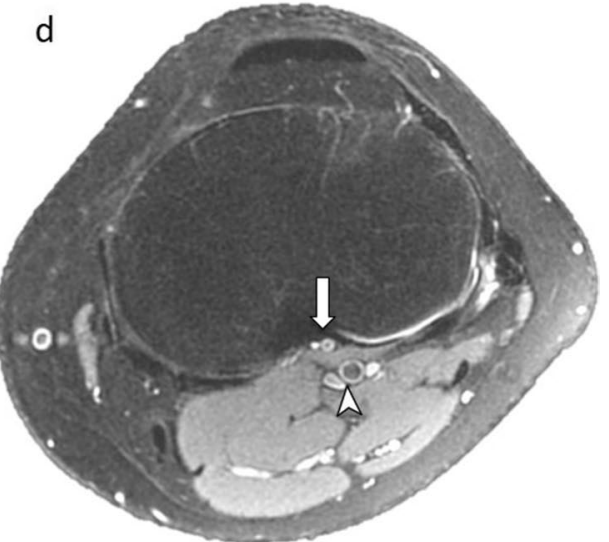

Fig. 1 a, b Schematic axial diagram of normal knee anatomy and Axial PDFS MRI of normal knee anatomy showing the popliteal artery (arrowhead) located posterior to the popliteal muscle (PM). c, d Schematic axial diagram and Axial PDFS MRI of the knee that show the aberrant anterior tibial artery (arrow) between the tibial cortex and the popliteal muscle (PM). Fibula (F), Gastrocnemius muscle media head (GMmh), Gastrocnemius muscle lateral head (GMIh). Figure created by J. Marin-Concha 
a

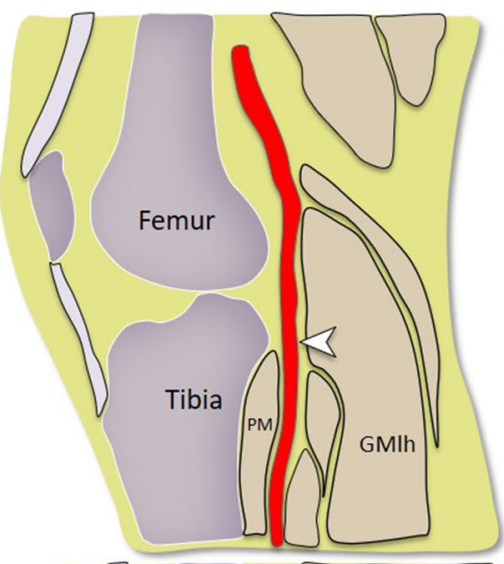

C

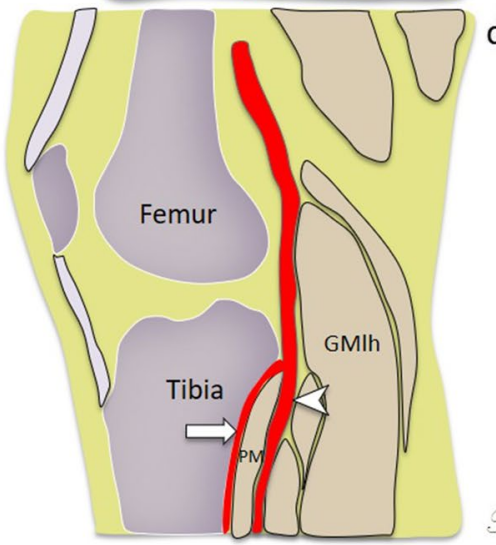

b

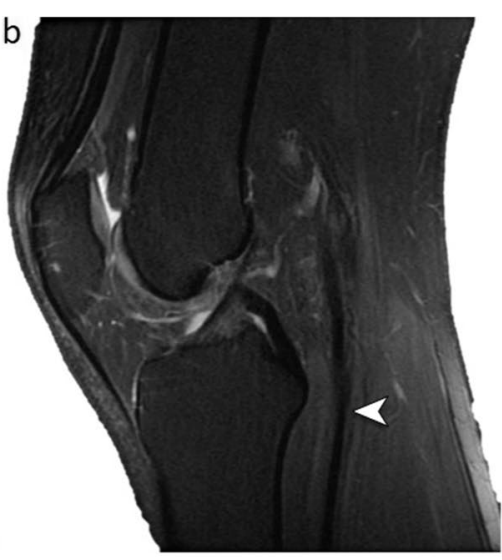

d

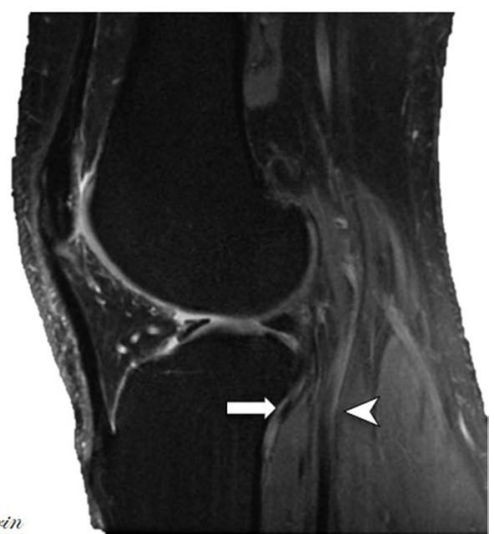

Fig. 2 a, b Schematic sagittal diagram of normal knee anatomy and sagittal PDFS MRI of normal knee anatomy show the popliteal artery (arrowhead) located posterior to the popliteal muscle (PM). c, d Schematic sagittal diagram and sagittal PDFS MRI of the knee that show the aberrant anterior tibial artery (arrow) between the tibial cortex and the popliteal muscle (PM). Fibula (F), Gastrocnemius muscle media head (GMmh), Gastrocnemius muscle lateral head (GMlh). Figure created by J. Marin-Concha

\section{Image acquisition and analysis}

Images were acquired on a 1.5 Tesla Optima MR450 and 1.5 Tesla Optima MR430s (GE Healthcare, Waukesha, Wisconsin, USA) using a dedicated 16 channel knee coil. The MRI protocol included acquisitions of proton density fat-saturated (PDFS) weighted images in coronal $(\mathrm{TR} / \mathrm{TE}=3100 / 35$; echo train length, $8 ; 3 \mathrm{~mm}$ thickness; space between slices $0.5 \mathrm{~mm})$, sagittal $(\mathrm{TR} / \mathrm{TE}=3300 / 35$; echo train length, $8 ; 2 \mathrm{~mm}$ thickness; space between slices $0.5 \mathrm{~mm}$ ) and axial (TR/TE $=3300 / 35$; echo train length, 8 ; $3.2 \mathrm{~mm}$ thickness; space between slices $0.5 \mathrm{~mm}$ ), coronal $\mathrm{T} 1$ weighted image $(\mathrm{TR} / \mathrm{TE}=600 / 12$; echo train length, 2; $3 \mathrm{~mm}$ thickness; space between slices $0.5 \mathrm{~mm}$ ) and sagittal T2 weighted images (TR/TE $=4360 / 90$; Echo train length, 20; $2 \mathrm{~mm}$ thickness; space between slices $0.5 \mathrm{~mm}$ ). Two experienced radiologists reviewed the knee MRI examinations and determined the presence or absence of this anatomical variation. Both radiologists were blinded to each other's findings. Cases of AATA were reviewed again by both radiologists for consensus.

The anatomical variant of AATA was classified as shown in Figs. 1 and 2.

\section{Scoping review}

We conducted a scoping review using Medline, PubMed and PubMed Central (PMC), Google Scholar, Scopus and Embase databases to identify studies examining the frequency of the AATA in the literature. Eligibile studies included those conducted via anatomical dissection, ultrasound, CT, angiography and MRI of unilateral and bilateral knees of cadeveric and individuals without knee pathologies. The literature search was performed by two reviewers (JM, PR) between February 2021-June 2021 including articles published from 1928 to 2020 in English. The following MESH terms were included in the approach using Boolean operators 
"AND" and "OR" in Pubmed: "Popliteal artery", "Knee", "magnetic resonance imaging", "angiography", "computer tomography", "Doppler ultrasound". Further details are shown in Supplement 1. Six hundred and seventy five studies were detected using the term Mesh and search limits. One hundred and twenty five abstracts were screened for elegibility, and duplicates were excluded manually. Reference lists of the selected articles were manually reviewed for additional articles. Ultimately, 20 studies provided information identifiying the frequency of AATA. Data were reported per the guidelines established by the Preferred Reporting Items for Systematic reviews and Meta-analyses extension for scoping reviews (PRISMA-ScR) [10]. The data extraction was performed by two reviewers (JM, PR). The data that was extracted included the following: year of publication, location of study, study design, type of study, level of evidence, patient demographics, journal type and funding. Data was synthesized for frequency of the AATA. Study quality was assessed using The Newcastle-Ottawa Scale (NOS) [11] for assessing the quality of nonrandomized studies in order to provide a measure of robustness of the current body of evidence on the AATA with a focus on: selection of the study groups (representativeness of exposed cohor, selection of non-exposed cohort, ascertainment of exposure, demonstration that outcome of interest was not present at start of study); comparability of the groups (adjust for the most important factors and other risk factors); and the ascertainment of either the exposure or outcome (Assessment of outcome, follow-up length and loss to follow-up rate). Good quality studies obtain 3-4 stars in the selection domain, 1-2 in the comparability domain and $2-3$ in the exposure domain, Fair quality studies obtain 2 stairs in the selection domain, 1-2 in comparatibility and 2-3 outcome domain. Finally poor studies obtain $0-1$ in all the domains.

\section{Statistical analysis}

We registered all data and performed descriptive statistics using Microsoft Office Excel 2016, Los Angeles, California, USA. The kappa test of agreement was used to determine agreement for the recognition of the AATA. A kappa value of 0.2 was defined as slight agreement; 0.21 - 0.40 as fair agreement; $0.41-0.60$ as moderate agreement; $0.61-0.80$ as strong agreement, and $0.81-1.00$ as almost perfect agreement.

\section{Results}

Patients

We reviewed 280 consecutive knee MRI examinations of 253 patients. The median age of the patients was 41 years (interquartile range 31-52), and 136 (53.8\%) were males. In total, we examined 147 (52.6\%) right and 133 (47.5\%) left knees with an evaluation of both knees in 27 patients.

\section{Magnetic resonance imaging study}

We were able to localize the popliteal vasculature in all cases. Proton density fat-saturated images provided the best contrast resolution between vasculature and the surrounding structures on visual inspection. Axial and sagittal PDFS allowed the identification of a vascular structure anterior to the popliteal muscle and posterior to the tibial cortex to confirm the diagnosis of AATA (Figs. 3 and 4).

An aberrant anterior tibial artery was visualized in 8 of the $280(2.9 \%)$ examinations using PDFS and in sagittal

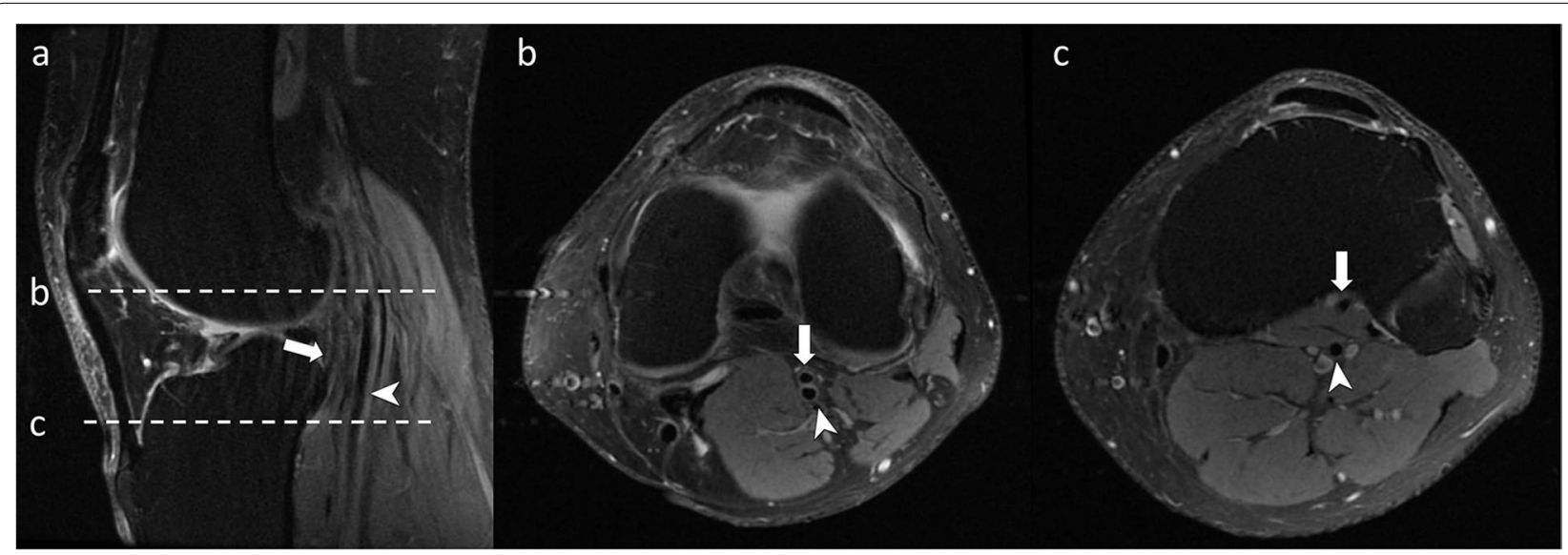

Fig. 3 A male patient (age range 45-65) with an aberrant anterior tibial artery. a Sagittal PDFS fat saturation shows the popliteal artery (arrowhead) running anteriorly to the popliteus muscle and the aberrant anterior tibial artery (arrow) posterior to the tibial cortex. b Axial PDFS image just below the origin of the aberrant anterior tibial artery. $\mathbf{c}$ Distribution of the aberrant anterior tibial artery and the popliteal artery at the upper part of the tibia. This is the typical location of a high tibial osteotomy surgery 


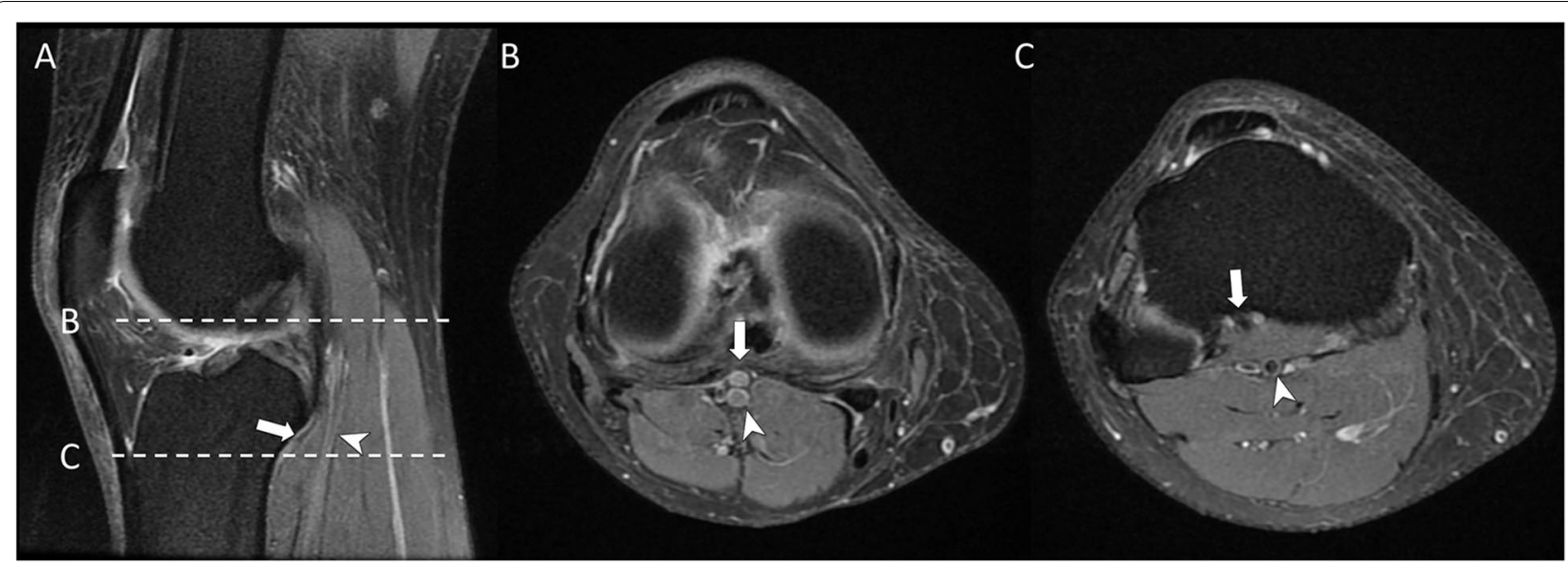

Fig. 4 A patient (age range 25-45) with an aberrant anterior tibial artery. a Sagittal PDFS shows the distribution of the popliteal artery (arrowhead) and the aberrant anterior tibial artery (arrow) that are separated by the popliteus muscle. $\mathbf{b}$ Axial PDFS image shows the origin of the aberrant anterior tibial artery. $\mathbf{c}$ Distribution of the aberrant anterior tibial artery and the popliteal artery at the upper part of the tibia

and axial orthogonal planes. The AATA was present in 5 cases in the left extremity and in 3 cases in the right extremity. In the 27 patients with MRI of both knees, the anatomical variant was present in 4 patients and was unilateral. In total, the prevalence of the aberrant anterior tibial artery was $3.2 \%(8 / 253)$ in our population. The images were evaluated with almost perfect agreement by the observers $(k=0.94)$. A second evaluation resulted in complete agreement regarding the final diagnoses.

\section{Literature review}

In our scoping review, we found 20 studies that evaluated and described the presence of the AATA by different methods. Figure 5 describes the identification and screening process used to create the final study list, which identified the studies, designs and study quality described in Table 1. Detailed results of study quality assessement are listed in Supplement 2. Three studies gathered data from limb dissection, 9 from angiograhy, 1 from ultrasound, 6 from CT angiography and 1 from MRI. The frequency of AATA ranged from 0.4 to $6 \%$ (median 1\%, IQR (0.5$2.3 \%)$. Some studies did not provide information about the gender, and 18 of the studies reported on additional anatomical popliteal artery variants besides the AATA.

\section{Discussion}

The main finding of our study is that in a Latin American cohort the AATA could be reliably detected using MRI displaying a relatively high prevalence of $3.2 \%$. This finding is closely similar to the frequency found in a previous angiographic study $(2.9 \%)$ and a cadaveric study (2.4\%) [7]. The only retrospective MRI study that determined the prevalence of AATA reported a frequency of $2.1 \%$ in an US-based population [9]. Notably, in the majority of our cases imaging studies were based on unilateral knee evaluation and therefore, bilateral assessment could yield higher prevalence of AATA. A subgroup of 27 patients that had MRI examination in both extremities had a prevalence of $14.8 \%$. This result was higher than previous retrospective angiographics studies that evaluated both extremities and reported lower prevalence $<3.2 \%$. Our results need to be interpreted with caution since the other studies that evaluated both knees enrolled a large number of patients.

Anatomical variants of the popliteal artery terminal branching are common. The final distribution of the popliteal artery is defined during the vascular embryologic period. The presence of the AATA is due to the persistence of the poplitea profunda artery and the failure in the development of the ramus communicans medius (Fig. 6) [21, 23, 24, 27, 28]. Historically, variation in the branching of the popliteal artery occurs in about $10 \%$ of patients [1, 29]. Lippert and Pabst [30] were among the first to classify the branching of the popliteal arteries according to the order of the splitting of the anterior tibial artery, posterior tibial artery and peroneal artery. Subsequently, Kim et al. [6] evaluated 605 femoral arteriograms and proposed a unified classification system of the popliteal arterial variants based on angiographic appearance (Type I: Normal Branching, Type II: high division of the poplietal artery and Type III: Hypoplastic or aplastic branching with altered distal supply). A subcategory of the high division of the popliteal artery, Type II, is when the tibial artery arises at or above the knee joint. If this vessel courses posterior to the popliteal muscle, it is called Type IIA1, but if 


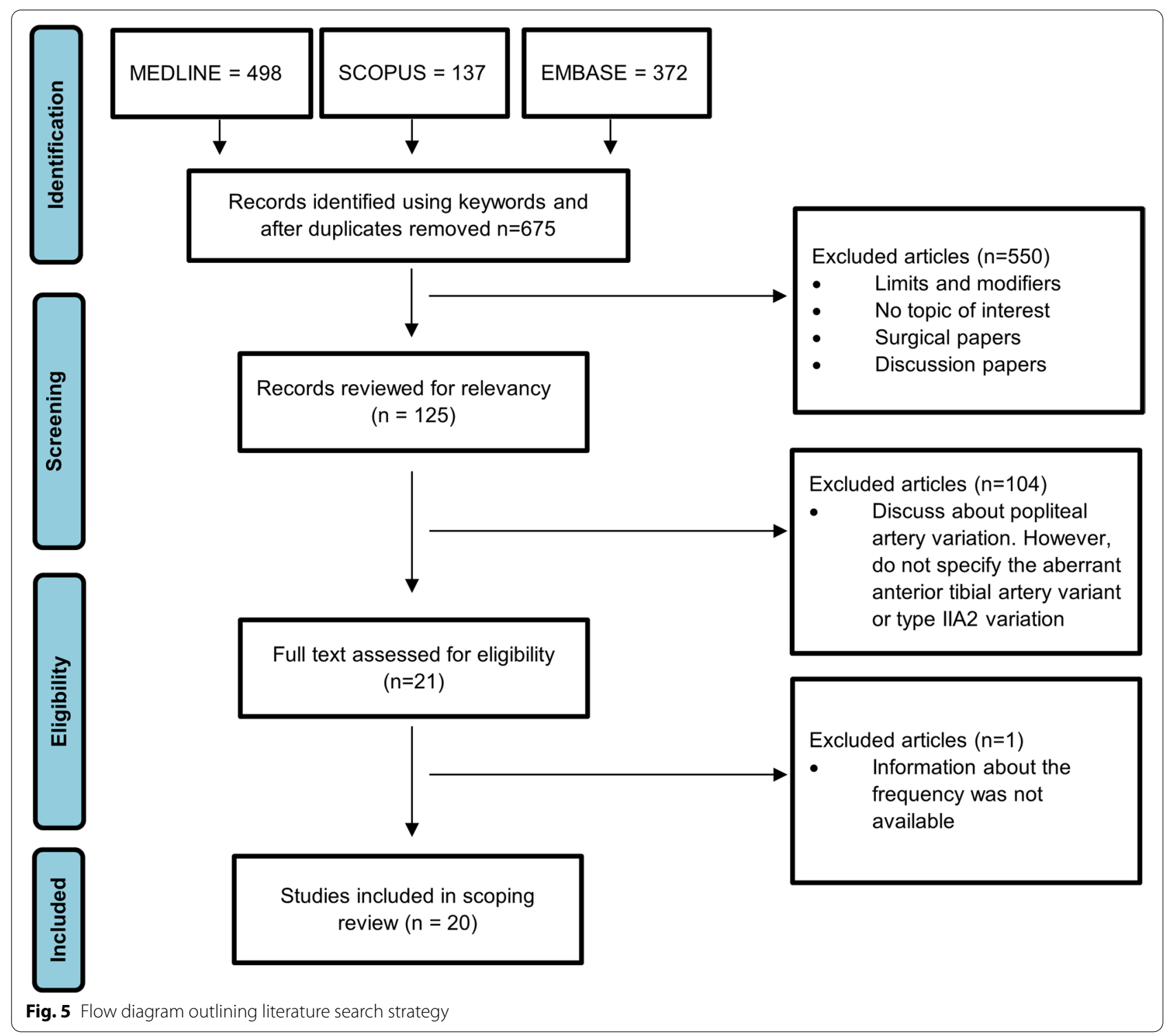

the anterior tibial artery courses between the anterior surface of the popliteal muscle and posterior tibial cortex, it is called Type IIA2 or AATA.

Cadaveric studies were among the first to report this anatomical variation $[17,30,31]$. The advantage of this method is that it allows a complete analysis of all the anatomical popliteal variants. A retrospective cadaveric study that evaluated 770 extremities in a Japanese population found that the AATA was present in $1 \%$ of the cases [31]. Another retrospective cadaveric study in a U.S population that evaluated 1168 extremities reported a frequency of $2.4 \%$ of the Type IIA2 variant [7]. The first imaging modality that was used to evaluate the popliteal branching was angiography. The presence of the AATA is suspected when the vessel takes a medial course initially before its common lateral course [6]. Different angiographic studies in European and South Korean populations reported frequencies that range from 0.4 to $2.9 \%$ $[12-16,18,29,32]$. Doppler ultrasound has been shown to be a precise method to evaluate the AATA despite its limitation assessing the entire knee vascular anatomy. A prospective study that evaluated 100 extremities using Doppler ultrasound showed that the prevalence of AATA was 6\% [20]. The advantage of CT angiographic over other methods is the complete and rapid identification of vascular and soft tissue structures. Most of the CT studies were retrospectives observational studies carried out in a European population showed frequency ranging 
Table 1 Frequency of aberrant anterior tibial artery in the literature

\begin{tabular}{|c|c|c|c|c|c|}
\hline Author & Methods & $\begin{array}{l}\text { Extremities } \\
\text { Examined }\end{array}$ & $\begin{array}{l}\text { Aberrant Anterior } \\
\text { Tibial Artery (\%) }\end{array}$ & Study design & $\begin{array}{l}\text { Quality } \\
\text { assessment } \\
\text { Newcastle- } \\
\text { Ottawa } \\
\text { Scale }\end{array}$ \\
\hline Adachi (1928) [12] & Limb Dissection & 770 & 1 & Retrospective observational & $5 / 9$ \\
\hline Trotter (1940) [7] & Limb Dissection & 1168 & 2.4 & Retrospective observational & $5 / 9$ \\
\hline Keen (1961) [13] & Limb Dissection & 280 & 0.4 & Retrospective observational & $5 / 9$ \\
\hline Kim (1989) [6] & Angiography & 600 & 0.7 & Retrospective observational & $5 / 9$ \\
\hline Davies (1989) [14] & Angiography & 200 & 1.5 & Retrospective observational & $5 / 9$ \\
\hline Prayer (1990) [15] & Angiography & 414 & 2.9 & Retrospective observational & $5 / 9$ \\
\hline Voboril (1990) [16] & Angiography & 253 & 0.4 & Retrospective observational & $5 / 9$ \\
\hline Day (2006) [17] & Angiography & 1037 & 2.4 & Retrospective observational & $5 / 9$ \\
\hline Szpinda (2006) [18] & Angiography & 152 & 0.66 & Retrospective observational & $5 / 9$ \\
\hline Kil (2009) [19] & Angiography & 1242 & 0.4 & Retrospective observational & $5 / 9$ \\
\hline Mavili (2011) [20] & Angiography & 535 & 1.3 & Retrospective observational & $5 / 9$ \\
\hline Celtikci (2017) [21] & Angiography & 1184 & 0.6 & Retrospective observational & $5 / 9$ \\
\hline Tindall (2006) [22] & Doppler Ultrasound & 100 & 6 & Prospective consecutive cohort & $5 / 9$ \\
\hline Yanik (2015) [8] & CT angiography & 126 & 4.4 & Retrospective observational & $5 / 9$ \\
\hline Calisir (2015) [23] & CT angiography & 742 & 0.5 & Retrospective observational & $5 / 9$ \\
\hline Oztekin (2015) [24] & CT angiography & 495 & 0.4 & Retrospective observational & $5 / 9$ \\
\hline Demirtas (2016) [25] & CT angiography & 1261 & 0.4 & Retrospective observational & $5 / 9$ \\
\hline Soler (2017) [11] & CT angiography & 1633 & 1.65 & Retrospective observational & $5 / 9$ \\
\hline Oner (2020) [26] & CT angiography & 340 & 0.6 & Retrospective observational & $5 / 9$ \\
\hline Klecker (2008) [9] & MRI & 1116 & 2.1 & Retrospective observational & $5 / 9$ \\
\hline Present cohort & MRI & 280 & 2.9 & Retrospective observational & \\
\hline
\end{tabular}

from 0.4 to $4.4 \%[8,19,22,25,26,33]$. In contrast with the previous imaging methods MRI appear as an excellent modality because it allows the complete analysis of the articular, vascular and soft tissue knee structures and is included in the mayority of presurgical knee evaluation. To our knowledge there is only one previous retrospective observational cohort that evaluated 1116 extremities using MRI showing a prevalence of $2.1 \%$. The study was done in an American population and reported that the AATA was most easily identified on axial and sagittal planes using PDWI [9]. We did not include this sequence in our knee MRI protocol institution, but we believe that sagitall and axial images are fundamental for the diagnosis.

Presurgical identification of anatomical variations such as the AATA can decrease the risk of vascular trauma [2, 34]. This is especially true in surgical orthopedic procedures such as knee joint surgery, total knee replacement, femoral distal or proximal tibial/fibular fracture surgery, high tibial osteotomy, lateral meniscal repair, posterior ligament cruciate reconstruction, and vascular interventions in popliteal artery aneurysm [5, 35]. Vascular injury can occur due to direct laceration, retraction and following the placement of screws during surgery $[3,4$, 36-41]. The region between the tibial cortex and major vessels (popliteal vein, popliteal artery and anterior tibial artery), also called "the danger zone", carries more than $20 \%$ of injury frequency during screw fixation in knee arthroplasty [41].

During orthopedic knee surgical procedures, the popliteal muscle has an important role because it protects the majority of vascular structures. However, in cases of AATA, the artery loses the protection of the popliteal muscle and is vulnerable due to the close relationship with the tibial cortex [35]. Interestingly, patients with a normal vascular distribution of the anterior tibial artery had a lower incidence of peripheral vascular disease than patients with an aberrant variation in a retrospective angiography study [16].

As a limitation our study did not evaluate both knees in the majority of cases due the fact that MRI is usually ordered to answer a specific problem. In our scoping review we found that $75 \%$ of the studies (angiographic and computed tomography studies) take into consideration both extremities showing no difference with the unilateral studies $[8,11,23-26]$. 


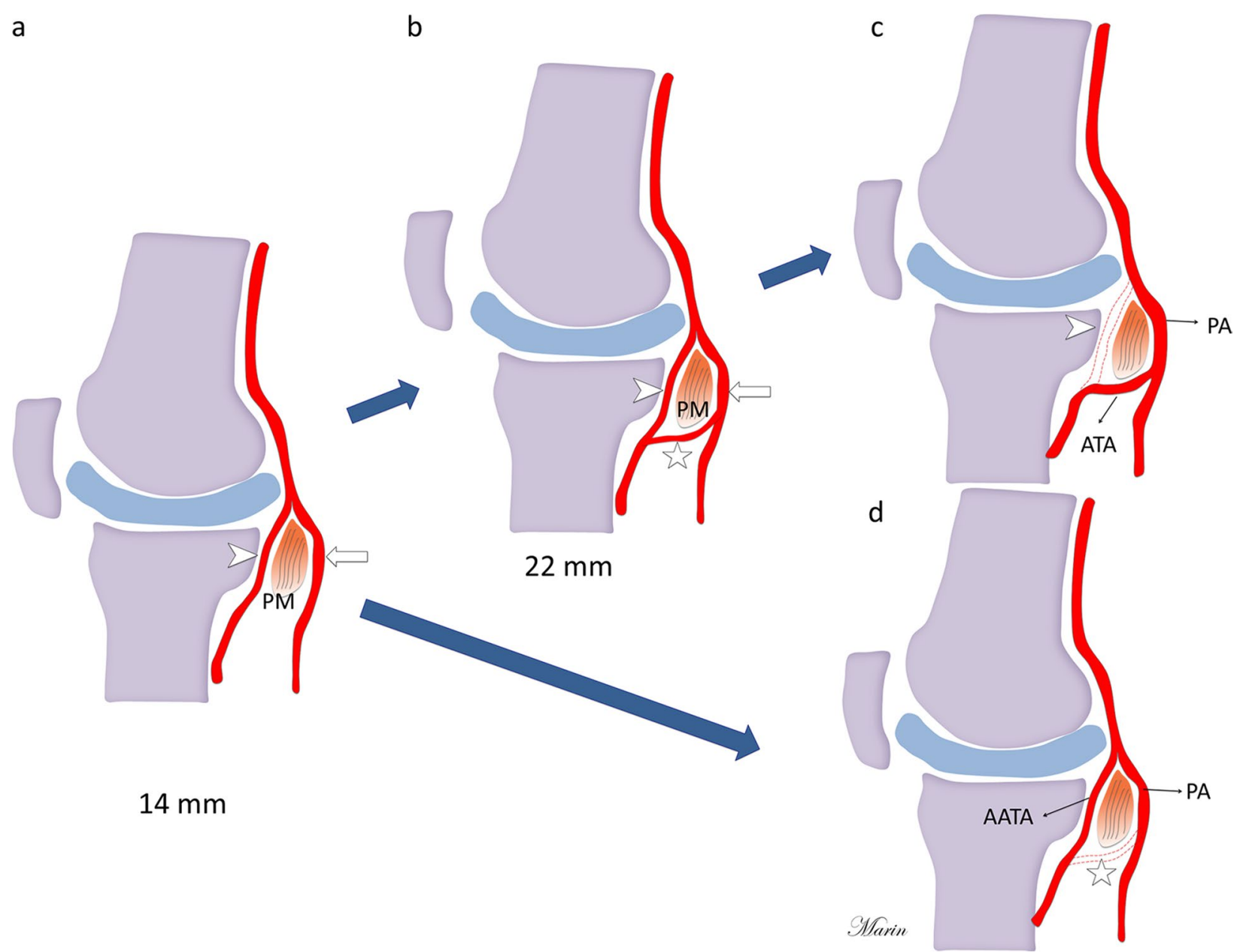

Fig. 6 Illustration of the embryological development of the aberrant anterior tibial artery. a By $14 \mathrm{~mm}$ the deep popliteal artery (arrowhead) courses anterior to the popliteal muscle (PM) and the superficial popliteal artery (arrow) lies posterior to the popliteal muscle. b By $22 \mathrm{~mm}$ a small connection between both arteries is formed and is called ramus communicans medius (star). $\mathbf{c}$ This vessel finally becomes the first part of the definitive anterior tibial artery (ATA) in normal cases. $\mathbf{d}$ Failure in the development of the ramus communicans medius will cause the persistence of the aberrant anterior tibial artery. Figure created by J. Marin-Concha

\section{Conclusions}

Precise anatomical characterization of the AATA using MRI is valuable in planning invasive interventional procedures of the lower leg since this anatomical variant was present in 8 out of 253 patients in our Latin American cohort. The prevalence of the AATA found in the included studies of the scoping review was $1 \%$ IQR (0.5-2.3\%).

\section{Abbreviations}

AATA: Aberrant Anterior tlbial Artery; MRI: Magnetic resonance imaging; PDFS: Proton density fat-saturated; TR: Repetition time; TE: Time to Echo; NOS: Newcastle-Ottawa Scale; CT: Computer tomography.

\section{Supplementary Information}

The online version contains supplementary material available at https://doi. org/10.1186/s12891-021-04801-9.

Additional file 1. Search Strategy Example: Ovid (Medline).

Additional file 2. Quality assessment (Newcastle Ottawa Scale).

\section{Acknowledgments}

This work is part of a Master's thesis of the Master's Program in Clinical Research, Center for Clinical Research and Management Education, Division of Health Care Sciences, Dresden International University, Dresden, Germany.

\section{Authors' contributions}

All authors conceived and designed the study. JMC, PR and PT analysed the data, JCM, DK and TS were major contributors in writing the manuscript. All 
authors revised the manuscript critically for important intellectual content and approved the final version to be published. TS supervised the study as principle investigator.

\section{Authors' information}

TS is program director of the Master's Program in Clinical Research, Center for Clinical Research and Management Education, Division of Health Care Sciences, Dresden International University, Dresden, Germany.

\section{Funding}

Open Access funding provided by Technische Universität Dresden. Dr. Siepmann received grants from the German Federal Ministry of Health, Kurt Goldstein Institut, German Parkinson Association, Michael J. Fox Foundation that were not related to this study. Dr. Siepmann received royalties from Astrazeneca for consulting and from Dresden International University for serving as program director and lecturer of the Master's Program in Clinical Research. Open Access funding enabled and organized by Projekt DEAL.

\section{Availability of data and materials}

The datasets used and/or analyzed during the current study are available from the corresponding author on reasonable request.

\section{Declarations}

\section{Ethics approval and consent to participate}

The need for ethics approval and informed consent was waived by the Institutional Review Boards of Clinica San Borja and the Universidad Peruana Cayetano Heredia due to the retrospective nature of the study. We obtained permission from Clinica SANNA, San Borja to get access to the clinical and imaging patient data.

\section{Consent for publication}

Not applicable.

\section{Competing interests}

The authors have no conflicts of interest to disclose.

\section{Author details}

${ }^{1}$ Radiology Department Clínica SANNA, San Borja, Lima, Peru. ${ }^{2}$ Division of Health Care Sciences Center for Clinical Research and Management Education Dresden, Dresden International University, Dresden, Germany. ${ }^{3}$ Institute of Diagnostic and Interventional Neuroradiology, University Hospital Carl Gustav Carus, Technische Universität Dresden, Dresden, Germany. ${ }^{4}$ Department of Neurology, University Hospital Carl Gustav Carus, Technische Universität Dresden, Dresden, Germany.

Received: 17 December 2020 Accepted: 19 October 2021

Published online: 02 November 2021

\section{References}

1. Kropman $\mathrm{RH}$, Kiela G, Moll FL, de Vries JP. Variations in anatomy of the popliteal artery and its side branches. Vasc Endovasc Surg. 2011;45(6):536-40.

2. Colborn GL, Lumsden AB, Taylor BS, Skandalakis JE. The surgical anatomy of the popliteal artery. Am Surg. 1994;60(4):238-46.

3. Langkamer VG. Local vascular complications after knee replacement: a review with illustrative case reports. Knee. 2001;8(4):259-64.

4. Daniels SP, Sneag DB, Berkowitz JL, Trost D, Endo Y. Pseudoaneurysm after total knee arthroplasty: imaging findings in 7 patients. Skelet Radiol. 2019;48(5):699-706.

5. Gaetke-Udager K, Fessell DP, Liu PS, Morag Y, Brigido MK, Yablon C, et al. Knee MRI: vascular pathology. AJR Am J Roentgenol. 2015;205(1):142-9.

6. Kim D, Orron DE, Skillman JJ. Surgical significance of popliteal arterial variants. A unified angiographic classification. Ann Surg. 1989;210(6):776-81.

7. Trotter $M$. The level of termination of the popliteal artery in the white and the negro. Am J Phys Anthropol. 1940;27(1):109-18.
8. Yanik B, Bulbul E, Demirpolat G. Variations of the popliteal artery branching with multidetector CT angiography. Surg Radiol Anat. 2015:37(3):223-30.

9. Klecker RJ, Winalski CS, Aliabadi P, Minas T. The aberrant anterior tibial artery: magnetic resonance appearance, prevalence, and surgical implications. Am J Sports Med. 2008;36(4):720-7.

10. Tricco AC, Lillie E, Zarin W, O'Brien KK, Colquhoun H, Levac D, et al. PRISMA extension for scoping reviews (PRISMA-SCR): checklist and explanation. Ann Intern Med. 2018;169(7):467-73.

11. Wells GA, Shea B, O'Connell D, Peterson J, Welch V, Losos M, et al. The Newcastle-Ottawa Scale (NOS) for assessing the quality of nonrandomised studies in meta-analyses. 2009. http://www.ohri.ca/programs/ clinical_epidemiology/oxford.htm. [cited 2009 Oct 19].

12. Prayer L, Karnel F, Schurawitzki H. Angiographic imaging of the branching of the popliteal artery into the arteries of the lower leg. Röntgenpraxis. 1990;43(3):100-3.

13. Voboril R. Note on variability of the arteries of the lower extremities in man. Folia Morphol (Praha). 1990;38(3):265-72.

14. Szpinda M. An angiographic study of the anterior tibial artery in patients with aortoiliac occlusive disease. Folia Morphol (Warsz). 2006;65(2):126-31.

15. Mavili E, Dönmez H, Kahriman G, Özaşlamacı A, Özcan N, Taşdemir K. Popliteal artery branching patterns detected by digital subtraction angiography. Diagn Interv Radiol (Ankara, Turkey). 2011;17(1):80-3.

16. Celtikci P, Ergun O, Durmaz HA, Conkbayir I, Hekimoglu B. Evaluation of popliteal artery branching patterns and a new subclassification of the 'usual' branching pattern. Surg Radiol Anat. 2017;39(9):1005-15.

17. Keen JA. A study of the arterial variations in the limbs, with special reference to symmetry of vascular patterns. Am J Anat. 1961;108:245-61.

18. Kil SW, Jung GS. Anatomical variations of the popliteal artery and its tibial branches: analysis in 1242 extremities. Cardiovasc Intervent Radiol. 2009;32(2):233-40.

19. Calisir C, Simsek S, Tepe M. Variations in the popliteal artery branching in 342 patients studied with peripheral CT angiography using 64-MDCT. Jpn J Radiol. 2015:33(1):13-20.

20. Tindall AJ, Shetty AA, James KD, Middleton A, Fernando KW. Prevalence and surgical significance of a high-origin anterior tibial artery. J Orthop Surg (Hong Kong). 2006;14(1):13-6.

21. Bolog NV, Andreisek G, Ulbrich EJ. MRI of the knee: a guide to evaluation and reporting. Switzerland: Springer International Publishing; 2015. p. 189-204.

22. Oztekin PS, Ergun E, CIvgın E, Yigit H, Kosar PN. Variants of the popliteal artery terminal branches as detected by multidetector ct angiography. Open Med (Warsaw, Poland). 2015;10(1):483-91.

23. Levinsohn EM, Hootnick DR, Packard DS Jr. Consistent arterial abnormalities associated with a variety of congenital malformations of the human lower limb. Investig Radiol. 1991;26(4):364-73.

24. Rohan A, Domagała Z, Abu Faraj S, Korykowska A, Klekowski J, Pospiech $\mathrm{N}$, et al. Branching patterns of the foetal popliteal artery. Folia Morphol (Warsz). 2019;78(1):71-8.

25. Demirtaş H, Değirmenci B, Çelik AO, Umul A, Kara M, Aktaş AR, et al. Anatomic variations of popliteal artery: evaluation with 128-section CT-angiography in 1261 lower limbs. Diagn Interv Imaging. 2016;97(6):635-42.

26. Oner S, Oner Z. Popliteal artery branching variations: a study on multidetector CT angiography. Sci Rep. 2020;10(1):8147.

27. Senior HD. Abnormal branching of the human popliteal artery. Am J Anat. 1929:44(1):111-20.

28. Olewnik Ł, Łabętowicz P, Podgórski M, Polguj M, Ruzik K, Topol M. Variations in terminal branches of the popliteal artery: cadaveric study. Surg Radiol Anat. 2019;41(12):1473-82.

29. Day CP, Orme R. Popliteal artery branching patterns -- an angiographic study. Clin Radiol. 2006;61(8):696-9.

30. Lippert H, Pabst R. Arterial variations in man: classification and frequency: Springer; 1985.

31. Adachi B. Das arterinsystem der Japaner. Band 1. A maxillaris interna; 1928. p. 85-96.

32. Davies B, Husami T, Lewis J, Retrum E. Developmental variations of the popliteal artery and its branches: a clinical correlation. Contemp Surg. 1989;34:28-32. 
33. Soler AG-C, Quiles-Guiñau L, Mata-Escolano F, Sanchis-Gimeno JA. Anatomic variations of the popliteal artery branches in present Spaniard population. Ital J Anat Embryol. 2017;122(3):206-15.

34. Yildiz S, Yazar F, Ozan H. A high-origin anterior tibial artery and its current clinical importance. Int J Anat Var. 2010;3:180-2.

35. Jang Y, Nguyen K, Rocky S. The aberrant anterior tibial artery and its surgical risk. Am J Orthop (Belle Mead, NJ). 2018;47(7). https://doi.org/10. 12788/ajo.2018.0057.

36. Padegimas EM, Levicoff EA, McGinley AD, Sharkey PF, Good RP. Vascular complications after total knee arthroplasty-a single institutional experience. J Arthroplast. 2016;31(7):1583-8.

37. Zhao R, LiY, Liu Y, Zhang K, Liu Z. Pseudoaneurysm of a high-division anterior tibial artery following primary TKA. Orthopade. 2017;46(3):275-9.

38. Chamseddin KH, Kirkwood ML. Anterior tibial artery pseudoaneurysm following ankle arthroscopy in a hemophiliac patient. Ann Vasc Surg. 2016;34:269.e17-.e19.
39. Salgado C, Mukherjee D, Quist M, Cero S. Anterior tibial artery pseudoaneurysm after ankle arthroscopy. Cardiovasc Surg. 1998;6(6):604-6.

40. Metzdorf A, Jakob RP, Petropoulos P, Middleton R. Arterial injury during revision total knee replacement a case report. Knee Surg Sports Traumatol Arthrosc. 1999;7(4):246-8.

41. Rubash HE, Berger RA, Britton CA, Nettrour WS, Seel MJ. Avoiding neurologic and vascular injuries with screw fixation of the tibial component in total knee arthroplasty. Clin Orthop Relat Res. 1993;(286):56-63. https:// pubmed.ncbi.nlm.nih.gov/8425368/.

\section{Publisher's Note}

Springer Nature remains neutral with regard to jurisdictional claims in published maps and institutional affiliations.
Ready to submit your research? Choose BMC and benefit from:

- fast, convenient online submission

- thorough peer review by experienced researchers in your field

- rapid publication on acceptance

- support for research data, including large and complex data types

- gold Open Access which fosters wider collaboration and increased citations

- maximum visibility for your research: over $100 \mathrm{M}$ website views per year

At BMC, research is always in progress.

Learn more biomedcentral.com/submissions 